\title{
Enhanced Energy Detector Via Algebraic Approach for Spectrum Sensing in Cognitive Radio Networks
}

\author{
Amira Ben Jemaa and Monia Turki \\ ENIT-U2S,University of Tunis El Manar \\ P.O.Box 37, Belvedere, 1002 Tunis, Tunisia \\ Email: Amira.BJ@gmail.com \\ Email:m.turki@enit.rnu.tn
}

\author{
Wael Guibène \\ EURECOM - Mobile Communication Dept \\ P.O.Box 193, 06904 Sophia Antipolis, France \\ Email: Wael.Guibene@eurecom.fr
}

\begin{abstract}
This paper ${ }^{1}$ deals with spectrum sensing techniques used to efficient utilization of limited spectrum resource. The most used technique is the energy detector as it is the simplest one for real time implementation and performs well for high SNR. This paper is concerned with this well known method and introduces an enhanced energy detector, in order to enhance its performance in low SNR. The proposed detector is based on the algebraic approach used for spike location, to make the detection more robust in a noisy environment. The proposed system model introduces an algebraic preprocessing bloc to attenuate the noise effect and enhance the detector performance at lower SNR. The detection is achieved by a second bloc that implements the conventional energy detection method. Simulation results, performed on DVB-T signals in additive Gaussian noisy context, show that the proposed detector performs much better than the conventional energy detector.
\end{abstract}

Index Terms-Cognitive radio, spectrum sensing algorithm, energy detector, algebraic detector, spike detection.

\section{INTRODUCTION}

The static frequency allocation scheme may not satisfy the increasing demand of the radio resource and may lead to an underutilization of spectrum resource. Many experimental researches [1] [2] show that the occupancy percentages of the spectrum radio may differ within frequency bands: some are so underutilized i.e. for a period of time and in a specific geographic point, an allocated frequency band may be unused by the licensed user.

An opportunistic spectrum sharing considers the possibility of the usage of this free space that appears as hole on the spectrum radio when the licensed user is not transmitting. For more efficient use of the spectrum resource, many research focus on cognitive radio to make possible a new dynamic frequency use. So that access to the vacant frequency band allocated to a primary user may be possible to unlicensed secondary user, for a period of time in a specific geographic point, avoiding any interference. In this concern, the main

\footnotetext{
${ }^{1}$ Author Wael Guibène wants to acknowledge the European Community's Seventh Framework Program (FP7/2007-2013) under grant agreement SACRA $\mathrm{n}^{\circ} 249060$ for founding his research activities.
}

challenge to make a such network scheme possible, is to detect the spectrum hole left by the primary user. the radio should achieve a spectrum sensing to classify the frequency band into vacant or occupied.

The main proposed techniques are the energy detector, the feature detector and the matched filter [3]. The optimal detection technique is the matched filter. However, it requires an a priori knowledge of the primary user signal features [4] which make the technique complex for implementation. Other methods based on cyclostationary features detection are proposed in [5][4]. The periodicity introduced in the signal format is used for detection. However, such technique still requires high computing performance. Compared to the matched filter and the cyclostationary feature detection, the energy based detection stills the less complex detector considering a real time context. It does not require any knowledge about the primary signal and is easy implemented. However, it suffers form the SNR wall problem [6].

We focus on a novel method introduced in [7][8] based on algebraic approach. This approach is based on the algebraic spike detection method used to detect spikes in EEG signal [9]. Since the presence of primary user can be shown on the spectrum amplitude by the presence of significant spikes in the occupied frequency band, this method can be applied to classify occupied bands and vacant ones. However, we obtain through the algebraic process a function highly correlated with the probability of the presence of spikes on the spectrum. This function can be considered as a smoothed version of the spectrum of the noisy input signal. The smoothing method based on the algebraic approach is different from conventional methods such as those based on spectral subtraction [11]. Indeed, it takes into account the specificity of the signal spectrum and also it does not require an estimate of the noise variance which can be a difficult task in noisy context. Based on this idea, we introduce an enhanced energy detector based on the energy detection and the algebraic approach in order to improve the performance of the detection in noisy environment.

The paper is organized as follows: Section II introduces 
the spectrum sensing problem. In section III, we review the algebraic approach for spike detection and emphasize its interest to smooth the spectrum of the noisy signal. We introduce, in section IV, our approach for a novel detector combining the algebraic approach and the energy detector, and propose an enhanced energy detector. Section V, deals with the performance evaluation of the proposed enhanced energy detector through simulation results.

\section{Spectrum Sensing Problem}

The main challenge for the cognitive radio is to detect the opportunity for available spectrum holes to access them for communication avoiding any interference with the licensed user. The detection of the presence of the licensed (primary) user can be modeled by a binary hypothesis testing problem :

$$
\left\{\begin{array}{lr}
H y p_{0}: y(n)=b(n) & \text { Vacant channel } \\
H y p_{1}: y(n)=s(n)+b(n) & \text { Occupied channel }
\end{array}\right.
$$

Assuming that $y(n)$ is the received signal by the cognitive radio, $s(n)$ is the signal emitted by the primary user and $b(n)$ is an additive white Gaussian noise.

The goal of the spectrum sensing is to decide about the availability of a frequency band for communication avoiding any interference.

Tow possible decisions can be made:

- $D_{0}$ when the cognitive radio decide that the sensed channel is vacant

- $D_{1}$ when the cognitive radio decide that the sensed channel is occupied

When the detected signal is considered as noise signal, the frequency band is then vacant and the cognitive radio can access it to communicate.

Two different wrong decisions can be made:

- The misdetection when a primary user is communicating so that the channel is occupied, however, it declares that the sensing area is a white free space. The probability of misdetection is defined as:

$$
P_{\text {misd }}=P\left(D_{0} \mid H_{1}\right)=1-P_{d}
$$

To avoid any interference with primary user, the probability of detection $P_{d}$ should be as high as possible.

- The false alarm when a primary user is not communicating so the channel is vacant, however, it declares the presence of the primary user. The probability of false alarm is defined as follows:

$$
P_{f}=P\left(D_{1} \mid H_{0}\right)
$$

To guarantee an efficient use of the spectrum resource and a possible dynamic access scheme, the probability of false alarm, according to the Neyman-Pearson criterion, should be kept as small as possible with a highest probability of detection.

\section{Frequency Algebraic Detector}

\section{A. Mathematical Background}

The spike detection algorithm was developed in [9] to detect spikes in EEG signals. Since the presence of primary users corresponds in the radio spectrum domain to the presence of significant spikes in bands occupied by each users, the algebraic method can be then used for spectrum holes detection as it was applied for temporal neural spike detection in EEG signal.

An algebraic detector for spectrum sensing was introduced in [7][8] applying the same algorithm to the spectrum of the received signal.

Let's assume that $Y(f)$ is the spectrum of the received signal $y(t)$. We assume that the input noisy spectrum is given in form of a piecewise regular signal:

$$
Y(f)=\sum_{i=1}^{K} \chi_{\left[f_{i-1}, f_{i}\right]}(f) p_{i}\left(f-f_{i-1}\right)+N(f),
$$

Where:

$\chi_{\left[f_{i-1}, f_{i}\right]}$ : Characteristic function of the interval $\left[f_{i-1}, f_{i}\right]$ $p_{i}(f)$ : Polynomial functions of order $\mathrm{N}-1$

$\left(f_{i}\right)_{i \in[1, k]}$ : Discontinuity points resulting from multiplying each $p_{i}$ by $\chi_{i}$

$N(f)$ : Additive noise.

Considering $s(t)$ the clean version of the signal transmitted by the primary user, the spectrum of $s(t)$ is modeled as :

$$
S(f)=\sum_{i=1}^{K} \chi_{\left[f_{i-1}, f_{i}\right]}(f) p_{i}\left(f-f_{i-1}\right) .
$$

Let's $B$ a bandwidth given such that there is at most one discontinuity point $f_{v}$ in each interval:

$$
I_{v}^{B}=[v, v+B], v \geq 0
$$

After some calculation considering the $N^{t h}$ order derivative, it is shown that the discontinuity point, in the interval $I_{v}^{B}$, is solution of the following equation [7][8]:

$$
\sum_{k=1}^{n+1}\left(\begin{array}{l}
N \\
k-1
\end{array}\right) f_{v}^{N-k+1} \varphi_{k}(f)=0
$$

where,

$$
\varphi_{k}(f)=\int_{0}^{+\infty} H_{k}(v) S(f-v)
$$

and

$$
H_{k}(f)=\left\{\begin{array}{l}
{\left[\frac{\left(f^{u-1}(B-f)^{N+k-1}\right)}{(u-1) !}\right]^{(N)}, 0 \leq f<B} \\
0 \text { Otherwise }
\end{array}\right.
$$

Note that $u$ is a constant higher than the derivative order $N$. In simulations, $u$ is fixed to $(N+1)$.

It is important to note that $\varphi_{k}(f)$ is the filtered of the input spectrum by the linear filter $H_{k}(f)$. 


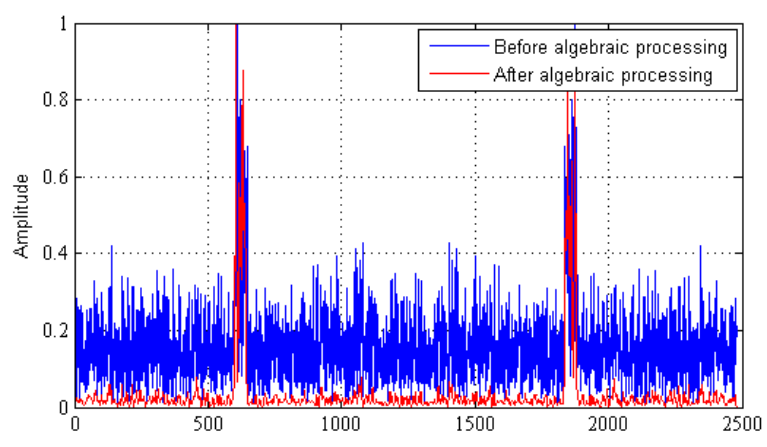

Fig. 1. Spectrum of the noisy signal $y(n)$ and the corresponding decision function of the algebraic detector $(\mathrm{SNR}=-5 \mathrm{~dB})$

$\left(\varphi_{k}\right)_{k \in[1, N+1]}$ are highly correlated with spectrum discontinuities locations. In absence of noise, except the "spike-like" frequency component $f_{v}, \varphi_{k}(f)=0$. When one $\varphi_{k}(f)=0$, all $\left(\varphi_{k}\right)_{k \in[1, N+1]}$ are also equal to 0 [9]. The decision function is then defined as:

$$
J(f)=\prod_{k=1}^{N+1} \varphi_{k}(f) .
$$

The presence of the primary signal in a specific band can be detected when $|J(f)|>0$.

However, the detector processes a noisy observation. The detection is then affected by less significant discontinuities relative to noise fluctuations. Consequently, the probability of presence of a discontinuity point $f_{v}$ is all the more high as $|J(f)|$ is high. A threshold must be set for final detection. It is fixed through Monte Carlo simulation. When only one channel band is scanned and since the totality of the spectrum band channel may be occupied by the signal, the spectrum may not represent significant spikes and therefore, the efficiency of the detector is very reduced. In fact, to ensure good performance, the detector must scan a set of $k$ adjacent channels where only $L<k$ channels may be occupied.

\section{B. Interest of Algebraic Approach as a Spectral Smoothing Process}

As shown in Fig.1, the signal output $J(f)$ (10) can be considered as the spectrum of a denoised version of the received signal spectrum. The algebraic approach performs as a smoothing process attempt to reduce the noise and extract the spikes shape on the spectrum.

The noise effect can be attenuated for a robust detection combining algebraic technique and any other spectrum sensing technique such as the energy detector since it performs well at high SNR but not at noisy environment.

The algebraic approach is used as a smoother preprocessing bloc, so the enhanced detector does not need to process a large frequency band and may consider only the observation over one channel.

As the algebraic detector processes the spectrum of the received signal through FIR filters, the algebraic smoothing can be then implemented in temporal domain by introducing algebraic analysis windows to avoid the Discrete Fourier Transform which may increase the detector complexity and introduce an additional time processing.

\section{ENHANCED ENERGY DETECTOR}

The proposed enhanced energy detector is based on algebraic approach as a preprocessing bloc combined with a conventional energy detector (Fig.2).

The algebraic preprocessing bloc is introduced as a temporal blind smoother bloc since it does not require any knowledge about noise or signal statistics.

\section{A. Temporal Algebraic Preprocessing Bloc}

Considering the spectral algebraic detector, the spectrum of the received signal $y(k)$ is processed by a filter bank structure composed of $(N+1)$ FIR filters which impulse response in frequency domain, are defined by $H_{k}$ (9).

Since the FIR filter $\left(H_{k}\right)_{k \in[1, N+1]}$ are independent of the input signal and in order to reduce complexity, we translate the processing in the time domain.

The received signal is consequently processed by temporal algebraic analysis windows $\left(h_{k}\right)_{k \in[1, N+1]}$ defined by,

$$
h_{k}(n)=T F D^{-1}\left(H_{k}(f)\right) .
$$

The output the preprocessing bloc $\phi(n)=T F D^{-1}(J(f))$ is then obtained as following:

$$
\Phi(n)=\phi_{1}(n) * \phi_{2}(n) * \ldots \phi_{N+1}(n),
$$

where $(*)$ denote the convolution operator and

$$
\phi_{k}(n)=H_{k}(n) \cdot y(n) \text {. }
$$

\section{B. Energy Detector Bloc}

The detection is then performed by a conventional energy detector. The detection of the presence of the licensed (primary) user can be modeled by binary hypotheses testing problem based on the signal output of the (algebraic) preprocessing bloc,

$$
\left\{\begin{array}{l}
\text { Hyp }_{0}: \Phi(n)=\tilde{b}(n) \\
\text { Hyp }_{1}: \Phi(n)=\tilde{s}(n)+\tilde{b}(n)
\end{array}\right.
$$

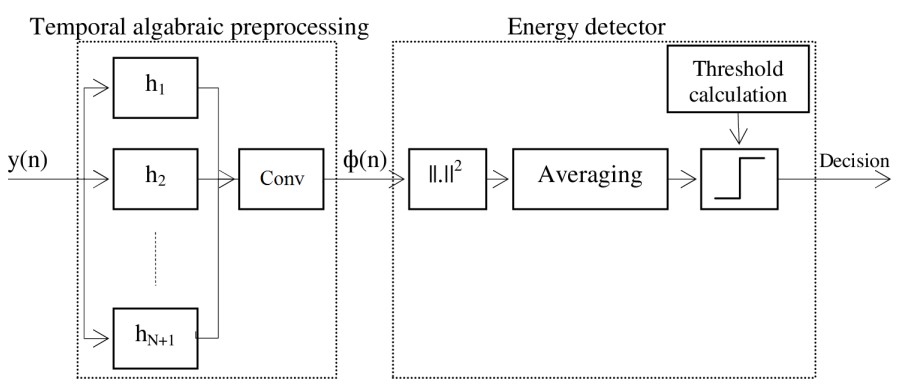

Fig. 2. Enhanced proposed Energy Detector Model 


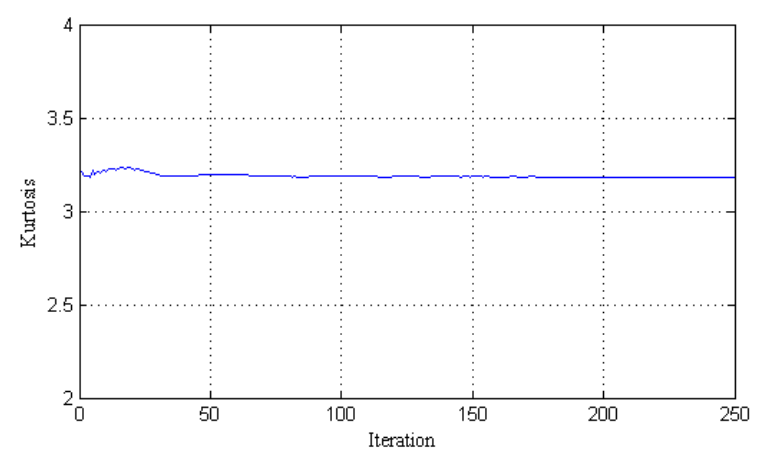

Fig. 3. Kurtosis function of the noise signal at the output of the preprocessing bloc (order $\mathrm{N}=2$ )

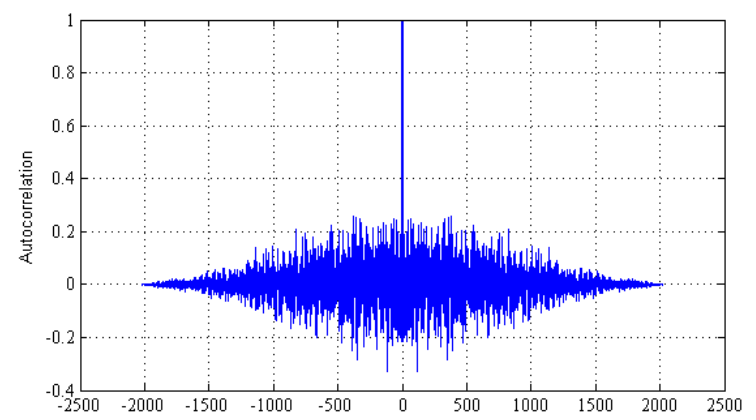

Fig. 4. Autocorrelation function of the noise signal at the output of the preprocessing bloc (order $\mathrm{N}=2$ )

The energy detector assumes that the noise is an additive white Gaussian noise.

Assuming that $b(n)$ is an additive white Gaussian noise, the noise $\tilde{b}(n)$ obtained at the output of the preprocessing bloc is assumed white Gaussian noise with variance $\sigma_{\tilde{b}}^{2}$ i.e $\tilde{b}(n)=$ $\aleph\left(0, \sigma_{\tilde{b}}^{2}\right)$. The white Gaussian noise hypothesis is, relatively, verified through simulation as shown in Fig.3 and Fig.4, where the kurtosis of $\tilde{b}(n)$ is very near to 3 and the autocorrelation function of $\tilde{b}(n)$ is similar to that of a white noise.

We denote $\tilde{s}(n)$ the signal component of $\Phi(n)$ The test static is then defined as:

$$
T=\frac{1}{M} \sum_{i=1}^{M-1} \Phi i^{2} .
$$

Where $M$ is the analyzed sequence length. The test static follows a chi-square distribution. When $M$ is sufficiently large, the test static can be approximated by a normal distribution as:

$$
T \approx \begin{cases}\operatorname{Normal}\left(\sigma_{\tilde{b}}^{2}, 2 \sigma_{\tilde{b}}^{4} / M\right) & \text { if } H y p_{0} \\ \operatorname{Normal}\left(\left(\sigma_{\tilde{b}}^{2}+\sigma_{\tilde{s}}^{2}\right), 2\left(\sigma_{\tilde{b}}^{2}+\sigma_{\tilde{s}}^{2}\right)^{4} / M\right) & \text { if } H y p_{1}\end{cases}
$$

The decision is then obtained by comparing the test static $T$ to a threshold $\gamma$. The probability of false alarm is defined for a threshold $\gamma$ as:

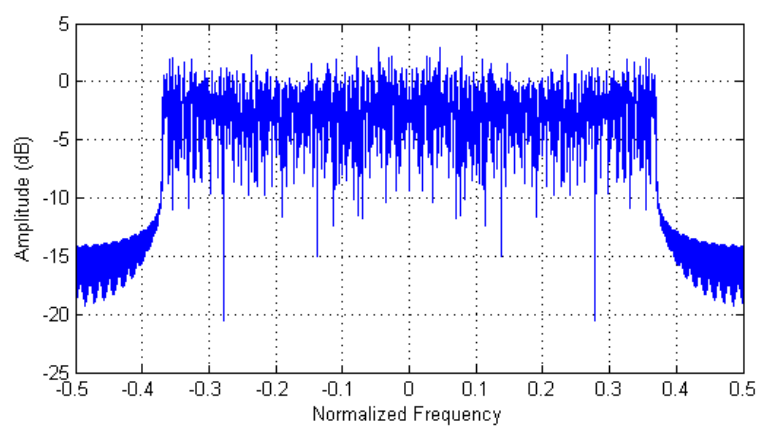

Fig. 5. FFT spectrum estimation of a DVB-T signal (Nfft=2048)

$$
P_{f}=P\left(T>\gamma \mid H y p_{0}\right) .
$$

The probability of false alarm is given by Marcum function as :

$$
P_{f}=Q\left(\frac{\gamma-\sigma_{\tilde{b}}^{2}}{\sigma_{\tilde{b}}^{2} / \sqrt{M / 2}}\right) .
$$

The threshold is then calculated to achieve a target probability of false alarm $\alpha$ as follows:

$$
\gamma=Q^{-1}(\alpha) \sqrt{2 \sigma_{\tilde{b}}^{4} / M}+\sigma_{\tilde{b}}^{2}
$$

\section{Simulation Results}

In order to evaluate the proposed enhanced energy detector, DVB-T signals are considered under AWGN channel.

Fig.6 shows the spectrum of a DVB-T signal at the input of the detector. The Gaussian noise is added to the DBV-T signal (Fig.5) according a Signal to Noise Ratio (SNR) $=5 \mathrm{~dB}$.

Fig.7 shows that the smoothing bloc succeeds to reduce the noise and preserve the specificity of the signal spectrum.

The probability of detection is evaluated for each SNR by Monte Carlo simulations. The detection is evaluated considering a threshold calculated to satisfy a required false alarm probability (19). The performance of DVB-T signal detection is illustrated in Fig. 8 with $P f=0.01$. We denote (AD k-ED) the proposed detector with $(\mathrm{k}+1)$ the derivative order.

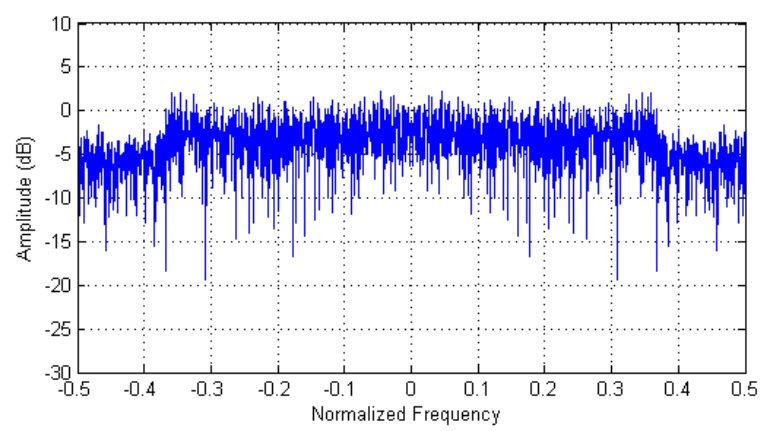

Fig. 6. FFT spectrum estimation of the signal at the input of the algebraic preprocessing bloc $(\mathrm{Nfft}=2048, \mathrm{SNR}=5 \mathrm{~dB})$ 


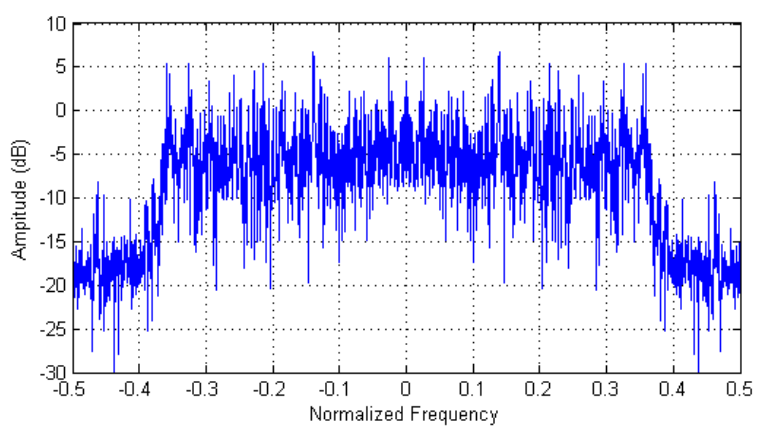

Fig. 7. FFT spectrum estimation of the signal at the output of the algebraic preprocessing bloc $(\mathrm{Nfft}=2048$, Order $\mathrm{N}=2, \mathrm{SNR}=5 \mathrm{~dB})$

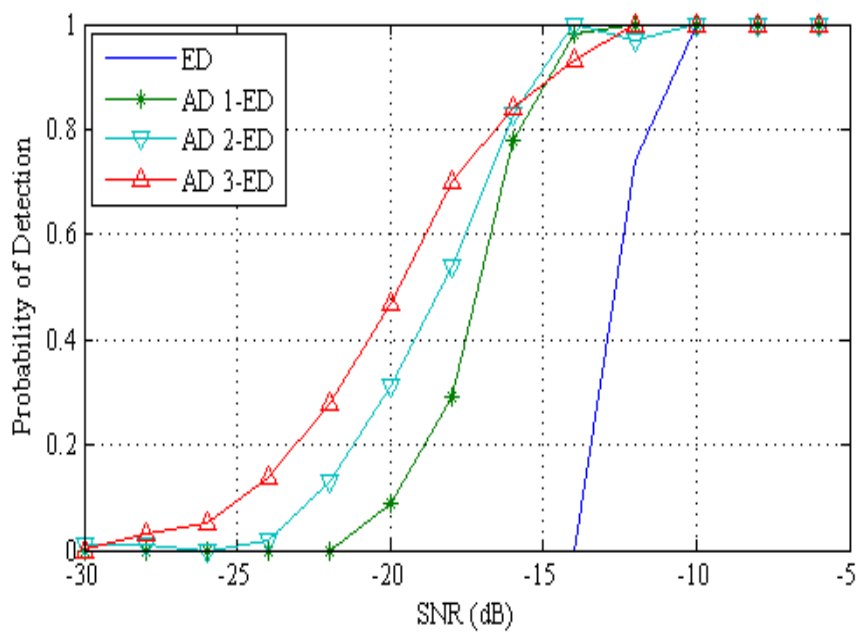

Fig. 8. Performance of the proposed enhanced detector (AD k-ED) for different orders, compared to the conventional energy detector (ED), $P_{f}=0.01$

The results show that the performance of the proposed enhanced energy detector is much better than the conventional energy detector. We notice a remarkable enhancement of the spectrum sensing technique based on energy detection which perform well at high SNR but cannot detect the presence of primary user for $S N R<-15 \mathrm{~dB}$. However, the proposed enhanced energy detector achieve a high probability of detection at $\mathrm{SNR}=-15 \mathrm{~dB}$.

A trade-off have to be made when choosing the detector order. As shown in Fig.8, the higher the polynomial order gets, the more preforming the detector gets, but the more complex the over-all system becomes.

\section{CONCLUSION}

In this paper, we focus on spectrum sensing technique based on algebraic approach. This approach was introduced for spike detection on the radio spectrum amplitude. The main challenge introduced by this approach is the choice of the decision threshold. However, the decision function obtained can be considered as a smoothed version of the received noisy signal. In order to enhance the use of this technique, we focus on this aspect to introduce a preprocessing bloc to attenuate the noise effect. We combine the algebraic preprocessing to the energy detector for a more robust detection. The preprocessing enhances the detection. The proposed detector achieves a higher probability of detection considering a very noisy environment.

\section{REFERENCES}

[1] M.Calabrese, "Broadcast to Broadband : Unlicenced Access to Unused TV Channels?", IEEE International computing, pp. 71-75, 2008.

[2] K.Steadman, D.Jones and Tugba Eprek, "Dublin Ireland Spectrum Occupancy Measurements Collected on April 16-18,2007", technical report shared spectrum company, 15 November 2007.

[3] M.Naraghipour and T.Lkuma, "A comparison of three classes of spectrum sensing techniques", IEEE Globecom Wireless Communication Symposium ,2008.

[4] Jarmo undén, "Specrtum sensing for cognitive radio and radar systems", Ph.D thesis, Helsinki university of technology, 2009.

[5] M.ghozzi, "Détection cyclostationnaire des bandes de fréquences libres", Ph.D thesis, University of Rennes I, 2008.

[6] J.Grosspietsch, Z.Ye and G.Memik, "Energy detection using estimated noise variance for spectrum sensing in cognitive radio networks", IEEE Wireless Communications and Networking Conference pp.711716, 2008.

[7] W.Guibène, M.turki, and A.Hayar, "Distribution discontinuities detection using algebraic technique for spectrum sensing cognitive radio networks", $5^{\text {th }}$ International conference on cognitive radio oriented wireless networks and communication, France, pp.1-5, June 2010.

[8] W.Guibène, M.Turki, B.Zayen and A.Hayar, "Spectrum sensing for cognitive radio exploiting spectrum sensing discontinuities detection", Published in EURASIP Journal on Wireless Communication and Networking, 2012.

[9] Z.Tiganj, M.Boup, C.Pouzat and L.Belkoura, "An algebraic method for eye blink artifact detection in single channel EEG recording", Proceedings $17^{\text {th }}$ International Conference on Biomagnetism Advances in Biomagnetism Biomag 2010, pp.175-178, 2010.

[10] J.O.Neel, J.H.Reed, Y.Zhao and S.Mao, "Performance evaluation of cognitive radios: Metrics, utility functions and methodology", Proceedings of the IEEE, pp.642-659, 2009.

[11] Y.Euphraim and D.Mallah, "Speech enhancement using a mean square error short-time spectral amplitude estimator", IEEE Trans. Acoust., Speech, Signal Processing, vol.32, pp. 1109-1121, 1984. 